\title{
Enfermagem e o tratamento medicamentoso da sífilis sob a ótica da Teoria Sócio-Humanista
}

\author{
Nursing and drug treatment of syphilis from the perspective of Socio-Humanist Theory \\ Enfermería y tratamiento farmacológico de la sifilis según la perspectiva de la teoría socio-humanista
}

Daniela Pollo' 'Q\%; Rogério Dias Renovato'/O

'Universidade Estadual de Mato Grosso do Sul, Dourados, MS, Brasil

\begin{abstract}
RESUMO
Objetivo: analisar o papel da enfermagem acerca da farmacoterapia da sífilis no âmbito da atenção primária em saúde. Método: investigação de abordagem qualitativa, com nove enfermeiros da atenção primária de município da Região Centro-Oeste do Brasil, realizada no período de agosto de 2018 a julho de 2019, por meio de entrevistas. A análise de dados apoiou-se na Teoria de Enfermagem Sócio-Humanista. Resultado: o papel da enfermagem esteve circunscrito à consulta de enfermagem, mediantes ações de acolhimento, escuta, detecção da sífilis, prescrição e administração de medicamento e práticas educativas. 0 aporte institucional caracterizou-se pela elaboração de protocolo sobre prescrição de medicamentos pelo enfermeiro e construção de fluxo de atenção ao usuário com sífilis. Conclusão: a enfermagem atua com autonomia na farmacoterapia da sífilis pautada em suas experiências e conhecimentos, aporte institucional, trabalho em equipe, procurando atender às necessidades de saúde do usuário.
\end{abstract}

Descritores: Teoria de Enfermagem; Enfermagem em Saúde Pública; Sífilis; Prescrição de Medicamentos.

\section{ABSTRACT}

Objective: to examine nursing's role in syphilis drug therapy in the primary health care context. Method: this qualitative study of nine primary care nurses in a municipality in Brazil's Midwest Region was conducted, by interview, from August 2018 to July 2019. Data analysis was based on the Social-Humanist Nursing Theory. Result: nursing's role was limited to nursing appointments, and took the form of welcoming reception, listening, syphilis detection, medication prescription and administration, and educational activities. The institutional contribution was characterized by preparation of a protocol on medication prescription by nurses and construction of the care flow for users with syphilis. Conclusion: nursing operates autonomously in syphilis drug therapy, seeking to meet user health needs based on its experience and knowledge, institutional support, and teamwork.

Descriptors: Nursing Theory; Public Health Nursing; Syphilis; Drug Prescription.

\section{RESUMEN}

Objetivo: examinar el papel de la enfermería en la terapia con medicamentos para la sífilis en el contexto de la atención primaria de salud. Método: este estudio cualitativo de nueve enfermeras de atención primaria en un municipio de la Región Medio Oeste de Brasil se realizó, por entrevista, de agosto de 2018 a julio de 2019. El análisis de datos se basó en la Teoría de Enfermería Social-Humanista. Resultado: la función de la enfermería se limitó a las citas de enfermería y se concretó en la recepción de bienvenida, la escucha, la detección de la sífilis, la prescripción y administración de medicamentos y actividades educativas. El aporte institucional se caracterizó por la elaboración de un protocolo de prescripción de medicamentos por enfermeras y la construcción del flujo de atención a usuarios con sífilis. Conclusión: la enfermería opera de manera autónoma en la terapia con medicamentos para la sífilis, buscando satisfacer las necesidades de salud de los usuarios a partir de su experiencia y conocimiento, apoyo institucional y trabajo en equipo.

Descriptores: Teoría de Enfermería; Enfermería de Salud Pública; Sífilis; Prescripciones de Medicamentos.

\section{INTRODUÇÃO}

A sífilis é uma infecção bacteriana causado pelo Treponema pallidum cuja transmissão se dá por via sexual, transplacentária (transmissão vertical) e transfusão sanguínea. A doença pode ser classificada, de acordo com o tempo de permanência no hospedeiro e sintomatologia, como sífilis primária, secundária, terciária e congênita (transmissão vertical), existindo também períodos de latência entre as fases ${ }^{1}$.

No Brasil, entre os anos de 2010 e 2019, totalizaram 650.258 casos de sífilis adquirida, e de 2005 a 2019, foram 324.321 casos de gestantes com sífilis. Entre 1998 e 2019, notificaram-se 214.891 casos de sífilis congênita. A maior incidência nas regiões brasileiras deu-se na região Sudeste, com os aumentos de 53,5\% de SA e $44,4 \%$ de SC, sendo que

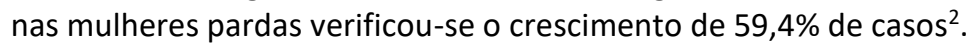

Agradecimento ao Programa Institucional de Iniciação Científica da Universidade Estadual de Mato Grosso do Sul (UEMS), ao Conselho Nacional de Desenvolvimento Científico e Tecnológico (CNPq) e ao Programa de Pós-graduação Ensino em Saúde, Mestrado Profissional (PPGES).

Autora correspondente: Daniela Pollo. E-mail: danielapollo93@hotmail.com

Editor responsável: Magda Guimarães de Araújo Faria 
No contexto do cenário atual da sífilis, o papel da enfermagem brasileira dispõe de atividades voltadas aos programas de saúde conduzidos pelo Sistema Único de Saúde (SUS) para o acompanhamento dos casos notificados. Conforme estabelece a Portaria no 2.488/2011, as atribuições do enfermeiro são especificamente direcionadas à realização de consulta de enfermagem, procedimentos, atividades em grupo, solicitações de exames complementares, prescrições de medicamentos e encaminhamentos, quando necessários, de usuários a outros serviços, estabelecidos conforme protocolos ou normativas técnicas ${ }^{1,3}$.

O papel da enfermagem no manejo da sífilis tem se ampliado para além da consulta clínica. Pelo Parecer no 26/2012, compete aos profissionais de enfermagem o direito de realizar teste rápido para detectar doenças como HIV, sífilis e outros agravos ${ }^{4}$. O protocolo das diretrizes clínicas de infecções sexualmente transmissíveis dispõe sobre a responsabilidade da solicitação de testes imunológicos para sífilis pelos enfermeiros ${ }^{5}$. Dessa maneira, a enfermagem desempenha atividades relacionadas à solicitação de testes, diagnóstico, prescrição de medicamentos e acompanhamento do usuário no tratamento da sífilis no âmbito da atenção primária à saúde (APS) ${ }^{6}$.

Acerca da penicilina G benzatina, a Decisão no 0094/2015, do Conselho Federal de Enfermagem (Cofen) e Nota Técnica Cofen/CTLN no 03/2017, reforça a importância do medicamento ao tratamento da sífilis e certifica a prática segura da administração pela equipe de enfermagem ${ }^{7,8}$. Diante da prescrição de medicamentos pelo enfermeiro, a Lei do Exercício Profissional n o 7.498 de 1986 e o Decreto Federal no 94.406 de 1987 asseguram a possibilidade de o enfermeiro prescrever medicamentos dentro dos programas do Ministério da Saúde, como o manejo da sífilis, desde que aprovado em normativas técnicas e protocolos seguidos de capacitações para tal ato ${ }^{9}$.

Entretanto, dentre as várias dificuldades apontadas pelos profissionais de enfermagem no enfrentamento da sífilis, está a prescrição da penicilina $\mathrm{G}$ benzatina, prática ainda centrada na atuação médica ${ }^{4}$. Em consequência disso, há baixas solicitações de testes e prescrições realizadas pelos enfermeiros, possivelmente em decorrência de insegurança e desconhecimento das possibilidades de suas práticas profissionais acerca da prescrição de medicamentos ${ }^{10}$.

Assim, o objetivo da pesquisa foi analisar o papel da enfermagem em relação ao tratamento medicamentoso da sífilis no âmbito da atenção primária em saúde sob a ótica da Teoria de Enfermagem Sócio-Humanista.

\section{REFERENCIAL TEÓRICO}

O referencial teórico fundamentou-se na Teoria de Enfermagem Sócio-Humanista (TESH), concebida pelas enfermeiras brasileiras Beatriz Beduschi Capella e Maria Tereza Leopardi, as quais aprofundaram os seus estudos em contexto social e administração institucional. A teoria envolve o indivíduo portador de doença, o profissional e a unidade de saúde ${ }^{11}$.

A teoria propõe a restauração e preservação da individualidade do sujeito portador de carência de saúde, considerando sua subjetividade e particularidades no processo do adoecimento. Também observa a necessidade da valorização do profissional para que possa reconhecer seus serviços e contribuir para a identidade da profissão e autonomia da assistência. Por conseguinte, a instituição tem como objetivo favorecer o desenvolvimento das relações entre o paciente e enfermeiro, garantindo os recursos e insumos necessários para assistência e segurança do tratamento ao usuário ${ }^{11}$.

\section{MÉTOdO}

Trata-se de um estudo exploratório, descritivo e qualitativo, realizado no município de Dourados - Mato Grosso do Sul, no período de agosto de 2018 a julho de 2019. No momento da pesquisa, o município contava com 88 enfermeiros atuando na secretaria municipal de saúde. Os critérios de inclusão da pesquisa foram ser enfermeiro atuando na APS e há pelo menos um mês no serviço. Não participaram da pesquisa, enfermeiros não atuando no momento da abordagem para entrevista por motivo de férias, licença médica e maternidade.

A amostra de nove enfermeiros deu-se por conveniência e pautada nos pressupostos da pesquisa qualitativa, em que as falas dos participantes são reveladoras do grupo em que está inserido, envoltas de dimensão sociocultural e das singularidades de seus tempos históricos ${ }^{12}$. A delimitação da amostra ocorreu por saturação, ou seja, ela aconteceu na medida em que os pesquisadores obtiveram maior proximidade ao objeto da pesquisa, evidenciando discursos que não acrescentavam dados novos à investigação ${ }^{12}$. Os convites para participação da pesquisa foram inicialmente para os enfermeiros das unidades de saúde, em que ocorriam as aulas práticas e estágios supervisionados curriculares obrigatórios do curso de enfermagem de universidade pública estadual.

As coletas foram obtidas por meio de entrevistas semiestruturadas e individuais de 15 a 30 minutos, agendadas previamente com os enfermeiros em seus locais de trabalho. As entrevistas semiestruturadas permitiam ao pesquisador obter informações solicitadas, concedendo também ao entrevistado a liberdade de elucidar suas experiências ${ }^{13}$. As 
entrevistas foram audiogravadas, datadas, transcritas e codificadas. Na primeira parte das entrevistas com questões fechadas, obtiveram-se informações sobre idade, sexo, instituição em que realizaram a graduação, tempo de serviço na APS, tipo de vínculo empregatício e pós-graduações realizadas. A segunda parte das entrevistas consistiu em perguntas abertas sobre o cotidiano desse profissional no manejo terapêutico da sífilis, suas experiências e vivências e suas percepções sobre o cuidado das pessoas e famílias, bem como a importância do seu papel como enfermeiro. 0 instrumento empregado nas entrevistas foi construído e elaborado pelos pesquisadores, sendo possível verificar sua abrangência em compreender as homogeneidades e diferenciações no grupo estudado ${ }^{12}$.

O referencial metodológico apoiou-se em Morse e Field, que se constitui em quatro etapas: apreensão, síntese, teorização e recontextualização. A apreensão decorreu dos registros das entrevistas, em que os conteúdos foram organizados e codificados. A síntese operacionalizou a análise dos relatos sobre a ótica da TESH, conhecendo e observando as experiências e rotinas dos entrevistados. A teorização buscou definir os relatos. A recontextualização obteve a interpretação das informações, alcançando o significado dos conteúdos analisados, contextualizando um novo saber para o objetivo da pesquisa sobre o papel do enfermeiro no tratamento medicamentoso da sífilis ${ }^{13}$.

A pesquisa foi encaminhada ao Comitê de Ética com Seres Humanos (CESH) da Universidade Estadual de Mato Grosso do Sul (UEMS), sendo aprovada com o parecer no 3.017.923. Para assegurar o anonimato, as falas dos enfermeiros foram codificadas por códigos Enf-1, Enf-2, Enf-3.

\section{RESULTADOS E DISCUSSÃO}

Da totalidade de enfermeiros entrevistados, sete eram do sexo feminino e dois do sexo masculino. Quanto à faixa etária, cinco estavam na faixa de 30 a 39 anos, três entre 40 e 49 anos e um entre 50 e 59 anos. Já quanto ao tempo de trabalho exercido dentro da APS, três trabalhavam entre 1 e 10 anos e seis tinham entre 11 e 20 anos de atuação na área. Todos disseram ter vínculo empregatício estatutário. Sobre os cursos de pós-graduação lato sensu, dez foram relatados, sendo que nove deles relacionados á saúde coletiva. Acerca de cursos de pós-graduação stricto sensu, uma enfermeira é mestre em Ensino em Saúde, e outra tem mestrado em Obstetrícia.

Evidenciou-se, a partir das falas dos enfermeiros, a relação dos vários aspectos do manejo terapêutico da sífilis, no âmbito da consulta de enfermagem, como a detecção da sífilis, a prescrição e administração do medicamento e as táticas de promoção para a adesão ao tratamento:

Aonde mais atuamos são nas consultas à gestante, porque na primeira consulta de pré-natal o enfermeiro realiza o teste rápido da sífilis e a detecta. Eu mesma posso prescrever o medicamento, solicito na farmácia. Então, desburocratizou. Eu posso ir lá pegar a medicação, posso prescrever e iniciar o tratamento (Enf-1).

As estratégias para o enfrentamento da sífilis pelo enfermeiro estão circunscritas tanto na consulta de enfermagem como em ações fora desse espaço de escuta e acolhimento. Dentre as ações, estão o rastreamento e controle de casos, que acontecem em campanhas e em programas de saúde, na propagação da educação em saúde, buscando a adesão ao tratamento e em todo o tratamento medicamentoso, desde a prescrição até administração e orientações sobre a terapêutica ${ }^{14}$. Na TESH, a assistência de enfermagem deve ser organizada em função das necessidades do usuário, visto que é dessa forma que o profissional consegue promover a compreensão dele sobre o tratamento, adequando-se à sua realidade social ${ }^{11}$.

O papel da enfermagem no tratamento da sífilis requer o aporte institucional para que suas ações sejam resolutivas. Desse modo, a construção de protocolo sobre prescrição de medicamentos pelo enfermeiro na APS elaborado e disponibilizado pela secretaria municipal de saúde e pela comissão de farmácia e terapêutica oportuniza práticas seguras e apoiadas no âmbito da gestão do serviço de saúde:

Depois que foi realizado o protocolo para a prescrição pelo enfermeiro, dentro da questão da sifilis, isto facilitou bastante para a gente. Temos, agora, um respaldo para prescrever, e não apresentamos dificuldades maiores com isso. Nós usamos o prontuário eletrônico; imprimimos a receita na hora e temos as medicações já guardadas aqui para a gestante iniciar o tratamento imediatamente. Como o protocolo pede, fazemos o teste VDRL, e depois comparamos com o teste final do tratamento (Enf-4).

A assistência depois do protocolo melhorou com certeza. Eu sinto a diferença. Eu sinto que nós temos uma resposta ao tratamento mais rápida porque como a primeira consulta é comigo, eu já início o tratamento. Então, eu vejo que isso é positivo (Enf-9).

Os protocolos vêm sendo utilizados para dar suporte às diretrizes do SUS, fomentando fluxos de cuidado mais sólidos aos enfermeiros. Sua implantação requer a contribuição de ações de educação permanente, provendo a participação dos profissionais enfermeiros, entendimento do processo de trabalho, apoio técnico e legal, e assim mais autonomia profissional ${ }^{15,16}$. Portanto, conforme a TESH, o papel atribuído à instituição é promover a construção de propostas direcionadas aos interesses e necessidades do usuário e do profissional, que permitam a autonomia profissional também no âmbito político, propiciando a qualidade da assistência e a valorização do trabalho ${ }^{11}$. 
A elaboração de fluxos de assistência no manejo terapêutico da sífilis em âmbito municipal favoreceu, além do acesso e prescrição de medicamentos, um itinerário do usuário mais simplificado e menos fragmentado, centralizando o cuidado nas unidades básicas de saúde:

Temos um controle agora, que é para relatar na ata. Por exemplo, este mês eu gastei 6 ampolas do medicamento [...] e levamos para a unidade básica de saúde (UBS) a ata como registro das pacientes. Como foram 3 pacientes com sífilis e são 2 frascos para cada, então lá ela me repõe. Antigamente eu tinha que pedir lá na CAF (Centro de Abastecimento Farmacêutico). Agora ficou mais fácil, pois descentralizou. A UBS é próxima e é fácil, pois eu posso passar lá e pegar. Bem diferente de antes (Enf-1).

Quando a gestão e os profissionais trabalham em conjunto para adequar o itinerário do usuário no SUS, fica aparente a preocupação em promover e demonstrar a ele o comprometimento em prover cuidado para a sua saúde. 0 acesso ao tratamento medicamentoso, configurado em protocolos, que se concretiza no fazer em saúde, além de trabalhar em prol da adesão ao tratamento, reduz a complexidade vivenciada pelo usuário quando busca a assistência à saúde na APS ${ }^{17,18}$. Na ótica da TESH, o trabalho em conjunto da instituição, como a Secretaria Municipal de Saúde, com o profissional enfermeiro é prerrogativa para atender às necessidades do usuário. A articulação instituição e profissional possibilita atentar para as relações subjetivas e sociais do cuidado em saúde e da enfermagem, amalgamada ao processo de trabalho em saúde ${ }^{11}$.

Para atuar no enfrentamento da sífilis, o profissional enfermeiro requer conhecimentos sobre a doença, prevenção e tratamento, além da habilidade em interpretar os dados dos exames, otimizando, assim, a terapêutica mais adequada para os usuários envolvidos:

Quando o teste do parceiro não dá regente, eu também faço o tratamento dele porque quando o teste rápido das gestantes deu positivo, entendemos que teremos que pensar no risco da criança de ter uma malformação [...] Dai tratamos o companheiro porque ele mantém relações sexuais com ela ou já teve ou poderá estar com uma janela sorológica. Nós pedimos o exame de sangue. É a titulação que vai dizer o quanto de lgM e lgG, $e$ nós nos baseamos nisso para iniciarmos o protocolo de tratamento junto com o parceiro, quando ele existe. Também é proposto o tratamento de terceiros envolvidos. Isso é sempre aberto na consulta (Enf-7).

A assistência do enfermeiro no manejo da sífilis consiste não apenas de conhecimentos técnicos e científicos, mas também de reflexão continuada sobre suas ações, que por vezes, produzem deslocamentos em como agir, ao considerar que as situações nem sempre são uniformes. Para isso, a prática da educação permanente é condição importante, que converge os saberes técnicos, as reinterpretações e ressignificações da sua prática profissional e a verificação de construção subjetiva como profissional de saúde ao lidar com situações que fogem do normal. Logo, por meio da "açãoreflexão-ação", o enfermeiro vai obtendo novos saberes provenientes de suas práticas profissionais, em busca de maior qualidade de seus serviços de enfermagem, qualidade que não é apenas técnica, mas perfilada pelas dimensões sociais e humanistas, conforme relata a TESH ${ }^{11}$

Dentre as ações do enfermeiro no tratamento medicamentoso da sífilis, o foco não se dá somente na prescrição e administração do medicamento, mas também no exercício das práticas educativas em saúde, em não apenas informar sobre, mas proporcionar a compreensão do uso do medicamento e de suas implicações:

Nós fornecemos os testes rápidos para a população [...] Se deu positivo, notificamos e iniciamos o tratamento. Nós falamos: "olha você vai tomar benzetacil". Então sabendo que o paciente não entende sobre a sífilis, então começamos a falar na linguagem em que ele compreenda (Enf- 2).

Ao adotar linguagens que fogem do vocabulário técnico, em geral, busca-se a fala simples e clara ao usuário, no ato da educação em saúde, na relação enfermeiro e usuário, demonstrando, assim, o acolhimento e empatia, fundamentada na concepção da equidiversidade. Logo, a comunicação efetiva proporciona a empatia e constrói o vínculo entre usuário e enfermeiro, colaborando com a adesão ao tratamento, como esclarece a TESH. Desse modo, o profissional enfermeiro configura-se também como educador em saúde, além de prescritor e responsável pela assistência ${ }^{11,19}$.

Dentre as estratégias relatadas pelos enfermeiros para sensibilizar os usuários com sífilis na adesão ao tratamento medicamentos, está o emprego de materiais didáticos, como desenhos e imagens relacionados à educação sexual:

Você tem que explicar todo o processo da sífilis. Eu faço desenhinhos e tudo: "olha a sífilis é uma doença que não é assim, depende de quem você tem relações sexuais. Com quantos no último ano? Esse que você teve relação no último ano, quantas relações vocês tiveram?". Eu fico desenhando porque é muito difícil de se falar "quem pegou de quem". Eu explico todo o processo: "O que é sifilis? É uma doença que se adquire através do sexo e é transmitida de pessoa para pessoa, se você estiver contaminado irá transmitir. Se você tratar e novamente estiver tendo relações sexuais, e o seu parceiro não tratar, irá se contaminar de novo" (Enf-2).

As estratégias de ensino-aprendizagem são utilizadas para oportunizar conhecimento e compreensão do estado de saúde do usuário. Caso o entendimento faça sentido para ele, espera-se que outras práticas sejam adotadas, decisões de tratamento sejam realizadas e a construção de novas rotinas saudáveis ocorram. A educação em saúde se amplia por meio 
de trocas de saberes, encorajando a autoanálise, reflexão, responsabilidade coletiva e a elaboração de novas concepções ${ }^{20}$. Na TESH, ressalta-se a importância de conhecer o paciente para que essas ações possam ser efetivadas da melhor forma. Assim, o conhecimento é entendido pelas subjetividades, crenças, culturas e vivências do sujeito. Dessa maneira, as estratégias de educação em saúde geram sentido, já que estão envolvidas na perspectiva de vida desse paciente ${ }^{11}$.

No âmbito da APS, o enfermeiro não atua solitariamente, mas em e com a equipe de saúde. 0 trabalho em conjunto da equipe da APS beneficia a aproximação das relações entre a comunidade e a unidade. Os agentes comunitários atuam no cadastramento da população, reconhecimento das condições socioeconômicas e sanitárias de cada família e realizam a busca ativa dos doentes, entre outras funções. Assim, as informações que os agentes comunicam aos enfermeiros sobre a população são cruciais para ações de campo de prognóstico, tratamento e prevenção ${ }^{21}$. O que vem convergir para os pressupostos da TESH, em que a assistência ao paciente é permeada de ações entre os trabalhadores de saúde, provendo estratégias colaborativas entre eles, com o intuito de enfrentar os agravos, como a sífilis, e assim proporcionar a assistência de enfermagem e de saúde mais resolutivas ${ }^{11}$.

Avisamos a própria parceira para que ele venha iniciar o tratamento, realizar os exames, e quando ele não vem, pedimos aos agentes de saúde realizarem a busca ativa. Registramos no prontuário, mas é difícil aquele que mesmo com a busca ativa não venha (Enf-4).

Um dos maiores desafios enfrentados pelos profissionais são a compreensão do companheiro sobre doença e o abalo da confiança do casal durante a detecção e a descoberta da sífilis:

Porque às vezes vem só a gestante e o parceiro não pôde vir porque trabalha, porque não quer. E muitos parceiros não querem [...] Então, eu grampeio na receita e faço uma receita só para o casal. Daí eu grampeio, a receita da penicilina na carteirinha da gestante e no verso da receita eu coloco as datas que eles devem tomar (Enf-8).

As descobertas da sífilis levam à instabilidade das relações amorosas, que são confrontadas dentro dos consultórios de enfermagem. Os enfermeiros vivenciam essas situações em seu cotidiano e, para isso, desenvolveram estratégias para acolher o casal por meio das práticas educativas em saúde, buscando a compreensão dos usuários envolvidos quanto à importância do tratamento para a saúde daquela família. Possivelmente não existe uma abordagem única ${ }^{22}$, mas cada profissional em sua trajetória vai elaborando métodos de proporcionar o entendimento da relevância em cumprir o tratamento, mesmo diante de situações, que podem envolver sentimentos de constrangimento e desconforto tanto para o profissional, como para os usuários. O tratamento medicamentoso da sífilis não se restringe apenas à detecção, prescrição e administração de medicamentos, e sim matizes complexos das relações humanas ${ }^{23}$.

Na representação gráfica da Figura 1, consta a síntese dos achados da pesquisa pautada em diagrama da TESH, trazendo o nível micro ou particular dessa rede de relações entre o ser humano (usuários com diagnóstico de sífilis) e enfermeiro, trabalhador em saúde, no âmbito da atenção primária.

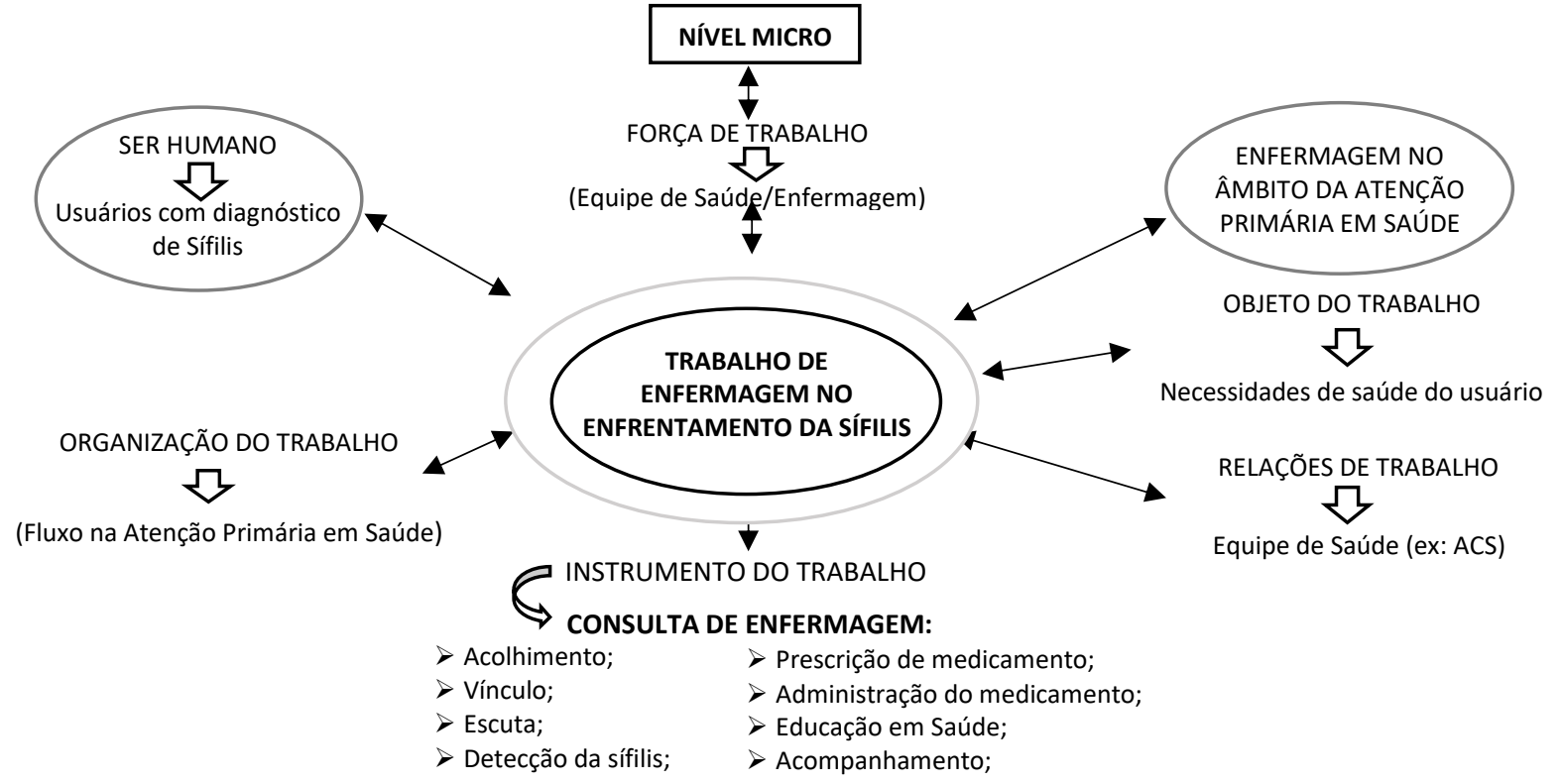

FIGURA 1: Nível micro de relação entre o ser humano, o trabalho de enfermagem no tratamento medicamentoso da sífilis e o seu meio, na ótica da Teoria de Enfermagem Sócio-Humanista. Dourados, MS, Brasil, 2019 
As ações do enfermeiro no enfrentamento da sífilis se materializam por meio da consulta de enfermagem, nas suas relações com a equipe de saúde, procurando atender às necessidades de saúde do usuário do SUS. Trata-se, portanto, de uma representação acerca da abordagem sócio-humanista, em contexto histórico particular, em que se estabelecem relações de subjetividade envoltas de sínteses complexas e contraditórias no processo de cuidar ${ }^{11}$.

\section{Limitações do estudo}

Como limitações do estudo, a pesquisa voltou-se para os profissionais de enfermagem e seu papel no tratamento medicamentoso da sífilis. Logo, é recomendável que outros estudos analisem as percepções dos usuários assistidos pelos enfermeiros em relação à sífilis, bem como os sentidos e significados da equipe de saúde quanto ao papel da enfermagem. Possivelmente, esses estudos poderão ampliar a compreensão do fenômeno estudado nesta investigação.

\section{CONCLUSÃO}

O papel da enfermagem em relação ao tratamento medicamentoso da sífilis na APS esteve circunscrito ao âmbito da consulta de enfermagem, mediante as ações de acolhimento, escuta, detecção da sífilis, prescrição e administração de medicamento, práticas educativas em saúde, com enfoque na compreensão da doença e na adesão ao tratamento.

O aporte institucional caracterizou-se pela elaboração de protocolo sobre prescrição de medicamentos pelo enfermeiro e construção de fluxo de atenção ao usuário com sífilis. O trabalho em equipe deu-se, principalmente, por meio da colaboração entre enfermeiro e agente comunitário de saúde.

As contribuições desta pesquisa para o campo da enfermagem e sua intersecção com o campo da saúde coletiva percorrem para além dos saberes e competências técnico-científicas do enfermeiro que atua na APS. Sob a ótica da TESH, construto teórico de autoria de enfermeiras brasileiras, ressaltam-se a valorização do ser humano em sua busca pelo cuidado à saúde; o reconhecimento do enfermeiro em sua prática que integra elementos objetivos e subjetivos do usuário, de si mesmo e da equipe na qual está inserido; e por fim, do papel da instituição, espaço em que o fazer da enfermagem torna-se concreto. Nesse campo de relações de cuidado, assistência e de trabalho em saúde, a proposta não é se conformar com o que está já delimitado, mas procurar brechas e proporcionar enfrentamentos persistentes em prol da humanização no cuidar.

\section{REFERÊNCIAS}

1. Nunes JT, Marinho ACV, Davim RMB, Silva GGO, Félix RS, Martino MMF. Syphilis in gestation: perspectives and nurse conduct. Journal of Nursing UFPE [Internet]. 2017 [cited 2020 May 26]; 11 (12): 4875-84. DOI: https://doi.org/10.5205/1981-8963v11i12a23573p4875-4884-2017.

2. Ministério da Saúde (Br). Secretaria de vigilância em saúde. Secretaria de atenção à saúde. Boletins, boletim de sífilis HIV/Aids/IST [Internet]. Brasília (DF): Ministério da Saúde; 2019. [cited 2020 May 26] Available from: http://www.aids.gov.br/pt-br/pub/2019/boletim-epidemiologico-sifilis-2019.

3. Ministério da Saúde (Br). Portaria № 2.488, de 21 de outubro de 2011 [Internet]. Brasília (DF): Ministério da Saúde; 2011. [cited 2020 set 21] Available from: http://bvsms.saude.gov.br/bvs/saudelegis/gm/2011/prt2488_21_10_2011.html.

4. Conselho Federal de Enfermagem (Cofen) [Internet]. Parecer normativo no001/2013. [cited 2020 May 26] Available from: http://www.cofen.gov.br/parecer-normativo-no-0012013_18099.html.

5. Ministério da Saúde (Br). Secretaria de Vigilância em Saúde. Departamento de Doenças de Condições Crônicas e Infecções Sexualmente Transmissíveis. Protocolo Clínico e Diretrizes Terapêuticas para Atenção Integral às Pessoas com Infecções Sexualmente Transmissíveis (IST) [Internet]. Brasília 9DF): Ministério da Saúde; 2020. [cited 2020 set 21] Available from: http://www.aids.gov.br/pt-br/pub/2015/protocolo-clinico-e-diretrizes-terapeuticas-para-atencao-integral-pessoas-cominfeccoes.

6. Camboim JCA, Sousa MNA, Lima CB, Silva MCL, Silva ANM, Camboim FEF. Prescrição de medicamentos por enfermeiros: legalidade, prática e benefícios. Rev Recien [Internet] 2017 [cited 2020 May 26]; 7(19): 15-27. DOI: https://doi.org/10.24276/rrecien2358-3088.2017.7.19.15-27.

7. Conselho Federal de Enfermagem (Cofen) [Internet]. Decisão no 0094/2015 [cited 2020 Sep 21] Available from: http://www.cofen.gov.br/decisao-cofen-no-00942015_32935.html.

8. Conselho Federal de Enfermagem (Cofen) [Internet]. Nota Técnica COFEN/CTLN no 03/2017 [cited 2020 Sep21] Available from: http://www.cofen.gov.br/wp-content/uploads/2017/06/NOTA-T\%C3\%89CNICA-COFEN-CTLN-N\%C2\%B0-03-2017.pdf.

9. Trindade Jr WB, Renovato RD, Sass GG. Manual educativo sobre boas práticas para prescrição de medicamentos pelo enfermeiro [Internet]. Produto técnico (mestrado) - Ensino em Saúde, Universidade Estadual de Mato Grosso do Sul, 2017. [cited 2020 May 26] Available from: http://www.uems.br/assets/uploads/cursos_pos/e147e39e86246f835839f40a04dc160b/teses_dissertacoes/1_e147e39e86246 f835839f40a04dc160b_2019-09-26_16-29-09.pdf.

10. Rodrigues DC, Domingues RMSM. Management of syphilis in pregnancy: knowledge and practices of health care providers and barriers to the control of disease in Teresina, Brazil. The International Journal of Health Planning Management [Internet], 2018 [cited 2020 May 26]; 33:329-44. DOI: https://doi.org/10.1002/hpm.2463. 
11. Capella BB, Leopardi MT. Teoria sócio-humanista. In: Leopardi, MT. Teorias de enfermagem: instrumentos para a prática. Florianópolis: Editora Papa Livros; 1999, p. 137-170.

12. Minayo MCS. Sampling and saturation in qualitative research: consensuses and controversies. Rev. Pesq. Qualitativa [Internet], 2017 [cited 2020 set 22]; 5(7), 01-12. ISSN 2525-8222. Available from: https://www.researchgate.net/publication/315756131_AMOSTRAGEM_E_SATURACAO_EM_PESQUISA_QUALITATIVA_CONSEN SOS_E_CONTROVERSIAS_SAMPLING_AND_SATURATION_IN_QUALITATIVE_RESEARCH_CONSENSUSES_AND_CONTROVERSIES.

13. Morse JM, Field PA. Nursing Research: the application of qualitative approaches. $2^{\text {nd }}$. edition. London: Chapman \& Hall; 1996.

14. Santana MVS, Barbosa GPN, Santos JFL. Sífilis gestacional na atenção básica. Div. Journ. [Internet] 2019 [cited 2020 May 26]; 4 (2), 403-19. DOI: https://doi.org/10.17648/diversitas-journal-v4i2.783.

15. Sales CB, Bernardes A, Gabriel CS, Brito MFP, Moura AA, Zanetti ACB. Standard operational protocols in professional nursing practice: use, weaknesses and potentialities. Rev Bras Enferm [Internet]. 2018 [cited 2020 May 26]; 71 (1): 126-34. DOI: http://dx.doi.org/10.1590/0034-7167-2016-0621.

16. Pereira JG, Oliveira MA. Nurses' autonomy in primary care: from collaborative practices to advanced practice. Acta paul. enferm. [Internet], 2018 [cited 2020 May 26]; 31(6):627-35. DOI: http://dx.doi.org/10.1590/1982-0194201800086.

17. Silva NEK, Freitas HAG, Sancho LG. From information's assimilation to men's therapeutic itineraries of men with or suspect of sexually transmitted diseases. The internet in focus. A internet em pauta. Physis [Internet] 2016 [cited 2020 May 26]; 26(2):66989. DOI: http://dx.doi.org/10.1590/S0103-73312016000200016.

18. Silva NEK, Sancho LG, Figueiredo W. Between flows and therapeutic projects: revisiting the notions of lines of care in health and therapeutic itineraries. Ciênc.saúde coletiva [Internet], 2016 [cited 2020 May 26]; 21(3):843-51. DOI: https://doi.org/10.1590/1413-81232015213.08572015.

19. Costa CV, Santos IAB, Silva JM, Barcelos TF, Guerra HS. Congenital syphilis: repercussions and challenges. Arquivos Catarinenses de Medicina [Internet]. 2017 [cited 2020 May 26]; 46(3): 194-202. Available from: http://www.acm.org.br/acm/seer/index.php/arquivos/article/view/94/191.

20. Fernandes JR, Silva VCF, Verissimo WP, Vianna T, Carneiro ML. Health education: the nurse's role as a health educator in IETC scenario. Rev. Jopic. [Internet]. 2019 [cited 2020 May 26]; 2 (04). Available from: http://www.revista.unifeso.edu.br/index.php/jopic/article/viewFile/928/670.

21. Domingues CC, Wandekoken KD, Dalbello-Araujo M. Potencialidades do processo de trabalho do agente Comunitário de Saúde. Rev. Bras. Pesq. Saúde [Internet]. 2018 [cited 2020 May 26]; 20(4):145-54. DOI: https://doi.org/10.21722/rbps.v20i4.24818.

22. Figueiredo MSN, Cavalcante EGR, Oliveira CJ, Monteiro MFV, Quirino GS, Oliveira DR. Perception of nurses on the adhesion of partners of pregnant women with syphilis to the treatment. Rev. Rene [Internet]. 2015 [cited 2020 May 26]; 16(3): 345-54. Available from: http://www.redalyc.org/articulo.oa?id=324041234007.

23. Silva CM, Santos NC, Afonso TM. Efetividade da assistência do enfermeiro da estratégia de saúde da família. Ciências Biológicas e da Saúde Unit [Internet]. 2018 [cited 2020 May 26]; 5(1):145-62. Available from: https://periodicos.set.edu.br/index.php/cadernobiologicas/article/view/5547. 